\title{
Control of Leakage Flow by Triple Squealer Configuration in Axial Flow Turbine
}

\author{
By Mohamed El-Ghandour, Mohammed K. IBrahIM, Koichi Mori and Yoshiaki NAKAMURA \\ Department of Aerospace Engineering, Nagoya University, Nagoya, Japan
}

(Received November 9th, 2008)

\begin{abstract}
A new turbine blade tip shape called triple squealer is proposed. This shape is based on the conventional double squealer, and the cavity on the tip surface is divided into two parts by using a third squealer along the blade camber line. The effect of the ratio of groove depth to span (GDS ratio) was investigated. The flat-tip case (baseline case) and doublesquealer case were calculated for comparison. In-house, unstructured, 3D, Navier-Stokes, finite volume, multiblock code with DES (Detached Eddy Simulation) as turbulence model was used to calculate the flow field around the tip. The computational results show that the reduction in the mass flow rate of the leakage flow for the triple squealer is $15.69 \%$ compared to the flat-tip case.
\end{abstract}

Key Words: Triple Squealer, Leakage Flow, Tip Clearance, Axial Flow Turbine, Internal Flow, CFD

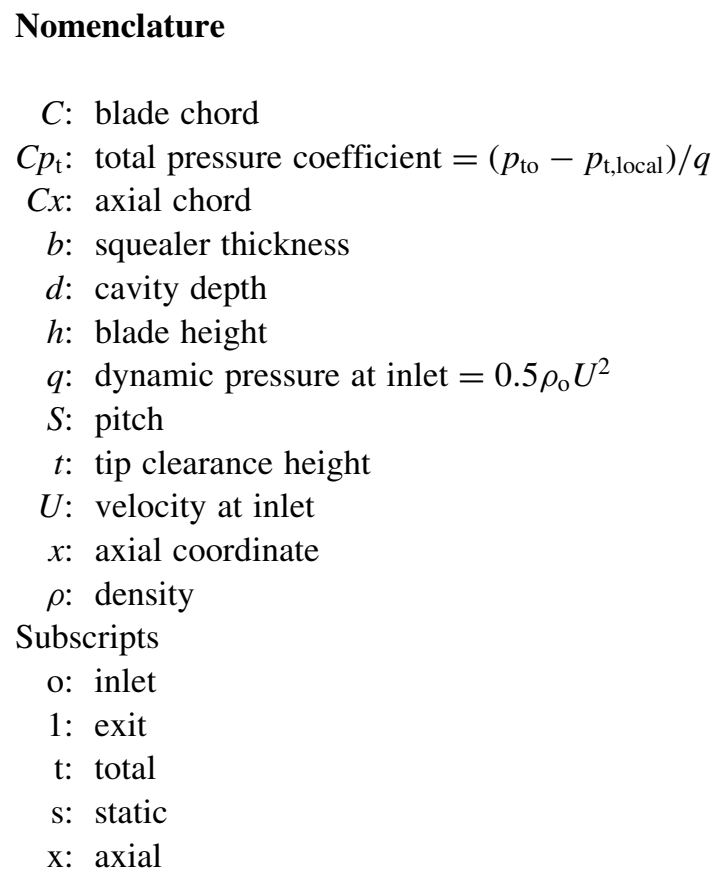

\section{Introduction}

Tip clearance is an essential feature of all turbomachines, allowing the relative motion between the blade tip and casing, preventing mechanical friction between them, and providing a suitable clearance for centrifugal and thermal expansions. On the other hand, due to pressure difference between the pressure and suction sides, tip clearance provides a path for flow escape from the former to the latter. In addition, the blade tip may burn-out or wear-out due to high heat transfer in the tip region. Incidentally, tip clearance accounts for up to one third of all losses in a blade row. ${ }^{1)}$

Many researchers have previously studied the tip leakage flow problem experimentally and analytically. Sjolander ${ }^{2)}$

C 2010 The Japan Society for Aeronautical and Space Sciences reviewed secondary and tip-clearance flows in axial turbines as well as their interactions. Recently, Bunker ${ }^{3)}$ made a concise and informative review of turbine blade functional, design, and durability issues. Control of the leakage flow by modifying the blade tip shape has been the main subject for much research. Azad et al. ${ }^{4)}$ studied the effect of squealer tip geometry on the heat transfer coefficient and static pressure distributions, examing six tip geometries. Their results showed that a squealer on the suction side provided better performance than on the pressure side or mid-camberline. Kwak et al. ${ }^{5)}$ extended the work of Azad to cover the tip and neighboring regions. Saxena et al. ${ }^{6}$ investigated the effects of a crosswise trip strip on the leakage flow and heat transfer rate. Cylindrical pin fins and pitch variation of strips over the tip surface were also investigated. Their results showed that trip strips placed against leakage flow reduced heat transfer on the tip by $10 \%-15 \%$ compared to a plain tip. Prakash et al. ${ }^{7)}$ proposed two blade tip geometries: a pressure-side tip shelf with vertical squealer tip wall, and a pressure-side tip shelf with inclined squealer tip wall. They found that the inclined wall reduced leakage and improved efficiency. Saha et al. ${ }^{8}$ examined the effect of a pressureside winglet on the flow and heat transfer over a blade tip. Their results showed that for a flat tip, the pressure-side winglet shows significant reduction in flow leakage. Recently Mischo et al. ${ }^{9)}$ proposed an improved design for a conventional recessed blade tip for a highly loaded axial turbine rotor blade. The improvement in overall efficiency was $0.2 \%$ in experiment and $0.38 \%$ in CFD.

Moore and Tilton ${ }^{10)}$ used a numeric potential flow model as well as a mixed model to simulate flow in the tip clearance of a linear turbine rotor blade cascade; the results gave better agreement with experiments. In addition, Moore et al. ${ }^{11)}$ used a 2D laminar flow model to investigate the effects of Reynolds number and Mach number on flow through a flat-tip clearance. Ameri and Steinthorsson ${ }^{12,13)}$ used a 3D, RANS model with an algebraic turbulence model 


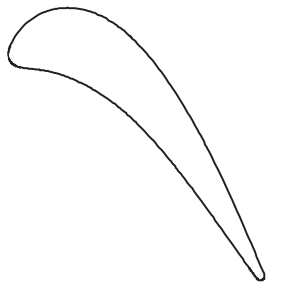

(a) Flat tip

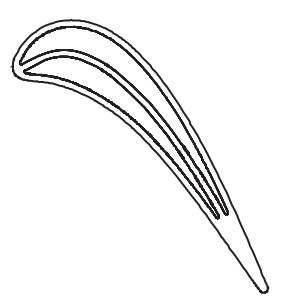

(c) Triple-squealer tip

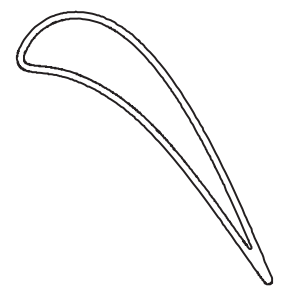

(b) Double-squealer tip

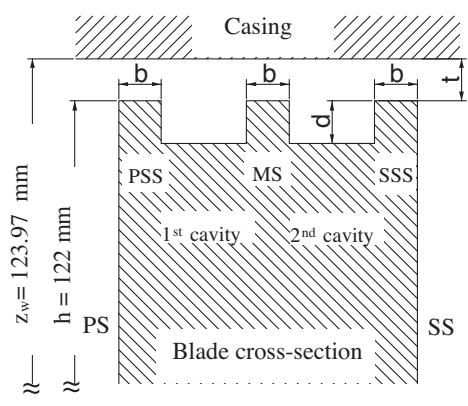

(d) Nomenclature and dimension of tip configuration
Fig. 1. Schematics of three tip geometries.

to predict the heat transfer rate on the tip of shrouded/ unshrouded turbine rotors of the SSME (Space Shuttle Main Engine). Yang et al. ${ }^{14)}$ applied three different turbulence models (standard high $R e k-\varepsilon$, RNG $k-\varepsilon$, and Reynolds stress model) to heat transfer prediction, and analyzed the heat transfer of the first-stage high-pressure turbine rotor blade of the General Electric-Energy Efficient Engine $\left(\mathrm{GE}^{\mathrm{B}} \mathrm{E}^{3}\right)^{15)}$

This paper proposes a new blade tip shape called triple squealer. This new shape is based on the conventional double squealer, but adds a third squealer along the blade camber line. Four cases for GDS ratio (ratio of groove depth to span): $0.75 \%, 1.5 \%, 2.25 \%$, and $3 \%$, which correspond to $50 \%, 100 \%, 150 \%$, and $200 \%$ of the tip clearance to span ratio (TCS ratio), respectively, were selected to examine the effect of GDS ratio on performance of the triple squealer. For comparison, the flat-tip case (baseline case) as well as double-squealer case were also calculated.

\section{Model in Study}

Figures 1(a)-1(c) show schematics of the three tip shapes used in this study. The tip nomenclature is shown in Fig. 1(d).

Calculation was performed for a three times scaled-up model in the same way as Yang et al. ${ }^{14)}$ This scaled-up model has an axial chord of $86.1 \mathrm{~mm}(C x=86.1)$, a span of $122 \mathrm{~mm}(h=122)$, and an aspect ratio of $1.4(A R=$ 1.4). The blade model is $2 \mathrm{D}$ with the same profile in the span direction. The tip clearance, $t$, is constant for all computations and is $1.5 \%$ of the blade span $(t=0.015 h)$, and the squealer thickness, $b$, is $2.3 \mathrm{~mm}$.

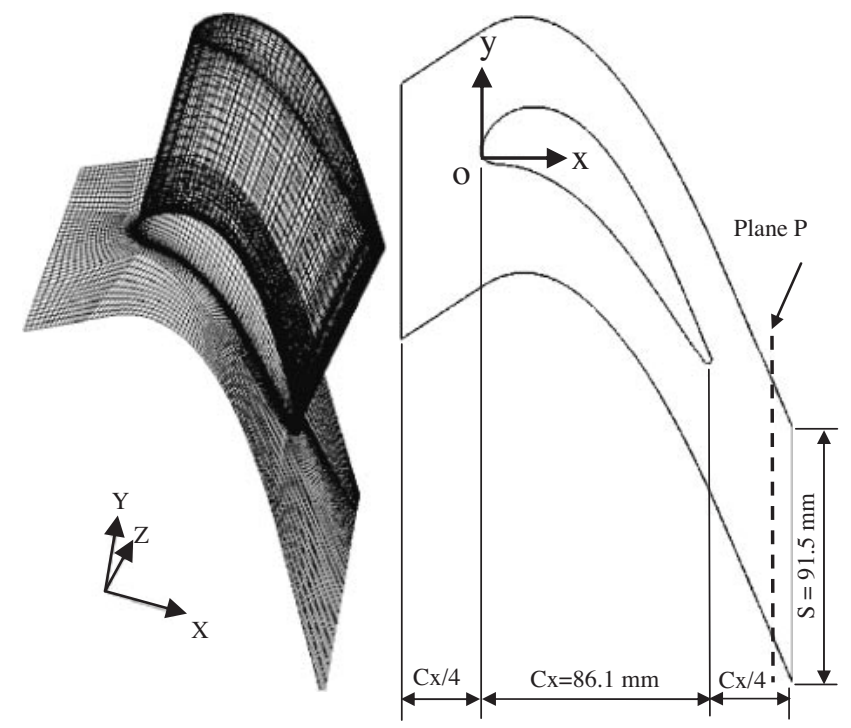

$\begin{array}{ll}\text { (a) 3D grid view } & \text { (b) Computational domain }\end{array}$

Fig. 2. Grid and computational region.

\section{Computational Method}

The numerical solver used here is in-house code. It uses an unstructured, finite volume, multiblock solver for the 3D compressible Reynolds-Averaged Navier-Stokes equations. Primitive variables on each side of a cell interface are interpolated using the 2rd-order MUSCL scheme with van Albada limiter, and inviscid numerical fluxes at the cell interface are calculated using Roe's approximate Riemann solver. The 2nd-order central differencing is applied for viscous numerical fluxes. The solution is advanced in time by LUSGS. For more details, refer to Kitamura et al. ${ }^{16)}$

In this study, this numerical code was modified for turbomachinery simulation by adding inlet boundary conditions regarding the total pressure and total temperature, and periodic boundary conditions regarding turbine blade cascade, as well as a subroutine to calculate the turbulent viscosity of DES based on the Spalart-Allmaras one equation turbulence model. ${ }^{17)}$ For boundary conditions, at the inlet plane, a total pressure of $129.96 \mathrm{kPa}\left(p_{\text {to }}=129.96 \times 10^{3}\right)$, a total temperature of $300 \mathrm{~K}\left(T_{\mathrm{to}}=300\right)$, and a flow angle of $32 \mathrm{deg}$. were given. At the exit plane, a static pressure of $108.3 \mathrm{kPa}\left(p_{\mathrm{s} 1}=108.3 \times 10^{3}\right)$ was applied. The no-slip and adiabatic boundary conditions were imposed at the wall; the periodic boundary condition was applied in the pitch direction. The casing wall was considered stationary.

The grid was generated first as a structured multiblock grid by using the commercial software Gridgen, and then modified to an unstructured grid. Figure 2(a) shows a 3D view of the grid used in this study. The computational domain for a single pitch of the GE- $\mathrm{E}^{3}$ (first stage rotor blade row) is shown in Fig. 2(b). The number of blocks and grid cells vary with the tip geometry. For example, for the triple-squealer case with a GDS ratio of $1.5 \%$, there are 507,009 grid cells distributed in 53 blocks, which includes 51,960 cells in the tip clearance and 17,920 cells in 


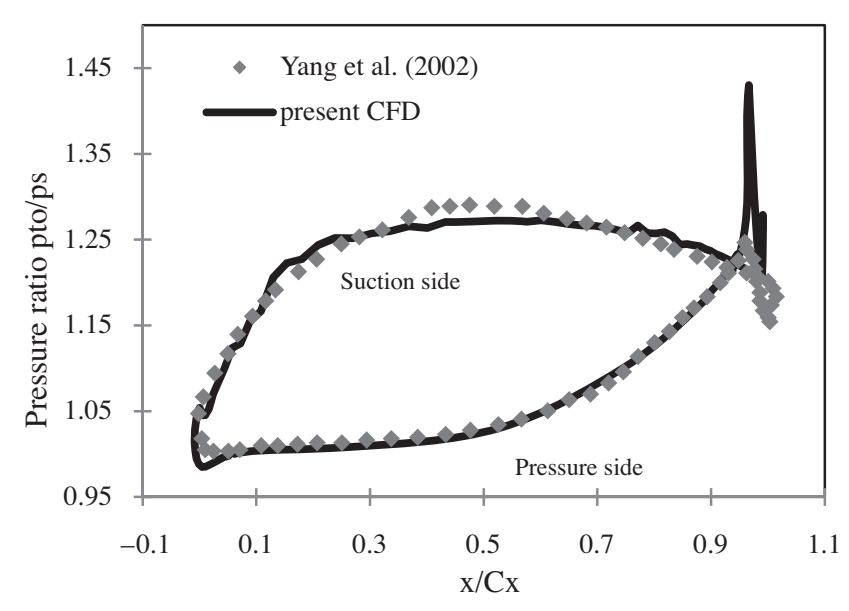

Fig. 3. Pressure distribution along blade surface at midspan section $(z / h=0.5)$ flat tip case.

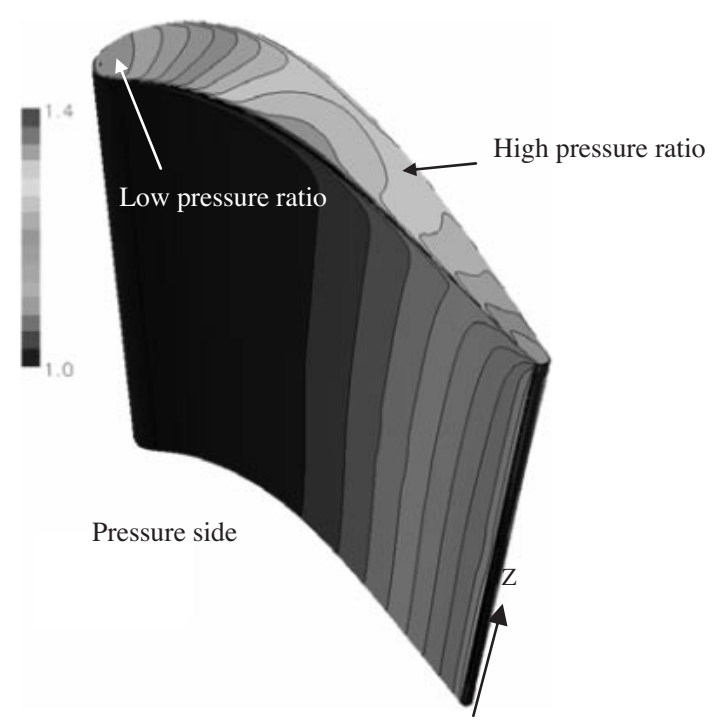

Fig. 4. Contours of pressure ratio, $p_{\text {to }} / p_{\mathrm{s}}$, on pressure side and tip surface (flat-tip case).

the cavities. The grid was clustered close to the blade and endwall surfaces. The first cell exists at $5 \times 10^{-6} \mathrm{~m}$ from the wall, which corresponds to $Y^{+}=1.47$.

\section{Results and Discussion}

\subsection{Validation of solver}

Figure 3 shows the pressure distribution along the blade surface in the case of the flat tip with TCS ratio of $1.5 \%$, along with the numerical results of Yang et al. ${ }^{14)}$ for validation. The vertical axis is the pressure ratio, $p_{\text {to }} / p_{\mathrm{s}}$, where $p_{\mathrm{s}}$ is the static pressure on the wall. Both results show reasonable agreement, except for some differences on the suction side and near the trailing edge, which is believed due to the difference in the turbulence model and grid topology.

\subsection{Flat-tip case}

The flow field and losses associated with the flat-tip case are described here as the study baseline. Figure 4 shows contours for the pressure ratio, $p_{\text {to }} / p_{\mathrm{s}}$, on the blade tip and pressure side.

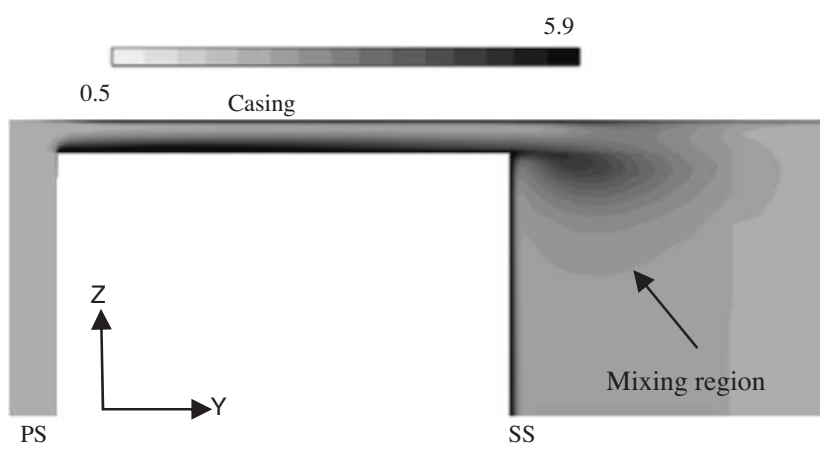

Fig. 5. Total pressure coefficient contours in plane with $x / C x=0.5$.

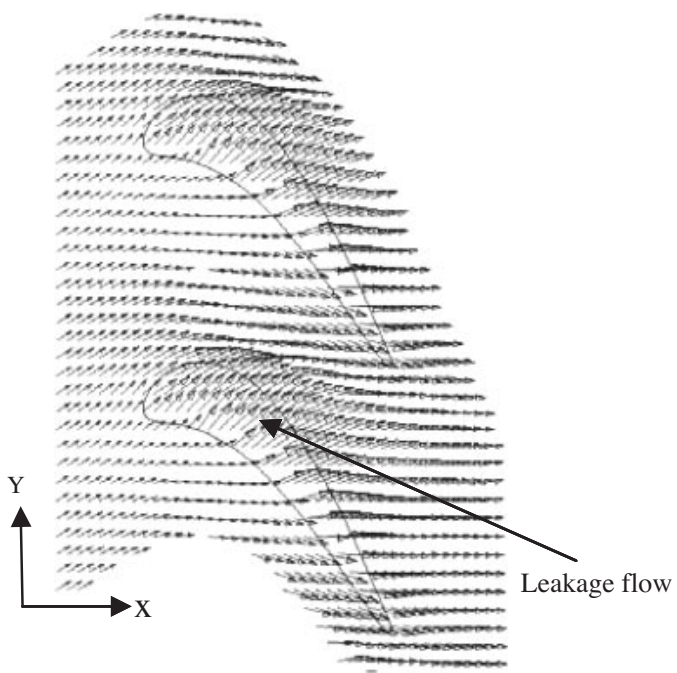

Fig. 6. Velocity vectors at mid-tip $(z-h) / t=0.5$.

The pressure distribution in the spanwise direction $(z-$ direction) is nearly uniform except for small deviations near the tip; other cases also show the same trend. This feature was previously noted experimentally by Sjolander and Amrud $^{18)}$ and numerically by Yang et al. ${ }^{14)}$ On the tip surface, there is a high pressure ratio (low static pressure) region, indicating a high velocity flow. This region covers about one third of the tip area. The flow direction is from the pressure side toward the suction side, which has an undesirable effect from the view point of efficiency and losses. In this case the maximum pressure ratio $\left(p_{\mathrm{to}} / p_{\mathrm{s}}=\right.$ 1.4) is observed close to the pressure side at $x / C x=0.47$. In addition, the average value of the pressure ratio on the tip surface is 1.245 .

The total pressure coefficient, $C p_{\mathrm{t}}$, represents the total pressure loss with respect to dynamic pressure at the inlet. Figure 5 shows the contours of the total pressure coefficient at plane $x=0.5 C x$. The high total pressure loss areas are mainly boundary layers and mixing zones.

Figure 6 shows the velocity vectors in a plane with $(z-h) / t=0.5$, which is between the tip and casing and parallel to the casing. From this figure that, flow close to the pressure side is entrained into the tip region and accelerated after entering the tip region. At the mid-chord region, the magnitude of leakage flow velocity is 3 to 4 times as 


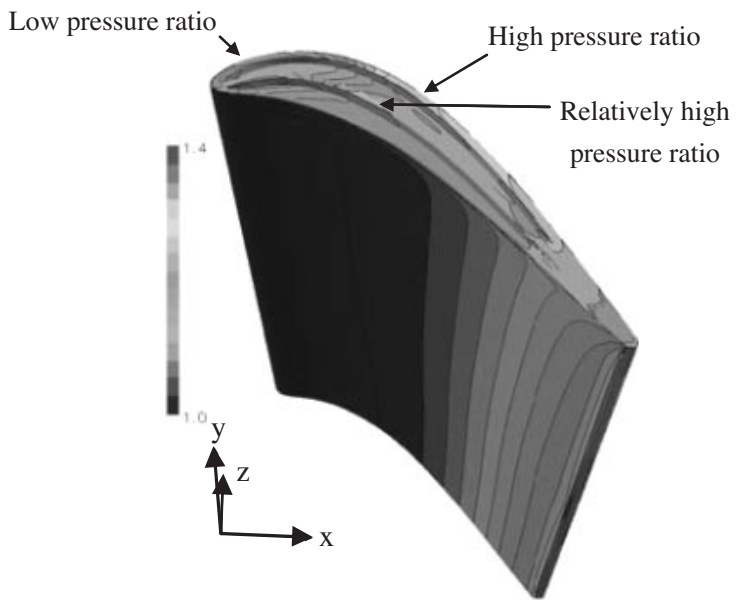

Fig. 7. Pressure ratio contours on blade-pressure side and tip for triplesquealer tip with GDS ratio of $1.5 \%$.

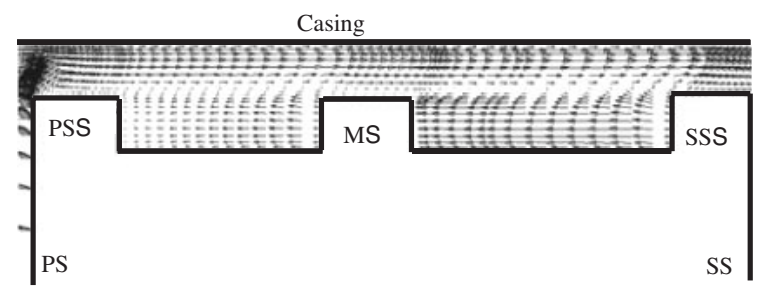

Fig. 8. Velocity vectors in plane with $x=0.5 C x$ (triple-squealer tip with GDS ratio of $1.5 \%$ ).

large as the inlet velocity. Even after it passes the tip, the flow maintains its direction and magnitude over some distance. This extension of the leakage flow into the main stream path reduces the available area for the main stream, and reduces the main stream mass flow rate. This finally reduces the output power of the machine.

\subsection{Triple-squealer case with GDS ratio of $\mathbf{1 . 5 \%}$}

The pressure ratio, $p_{\text {to }} / p_{\mathrm{s}}$, distribution on the triplesquealer tip with GDS ratio of $1.5 \%$ is shown in Fig. 7. The pressure distribution on the blade tip has changed dramatically. The high pressure ratio region at the mid-chord is reduced considerably, except in a small region at the middle of the suction side squealer. An almost uniform flow pattern is observed at the pressure-side squealer. The lowpressure ratio region near the leading edge is expanded and shifted from the suction side to the pressure side and is confined to the second cavity (close to suction side). There is also a relatively high velocity spot at the middle of the middle squealer.

Figure 8 shows the component of velocity $\left(v_{y}, v_{z}\right)$ in a cross-section $(x=0.5 C x)$, for triple-squealer tip with GDS ratio of $1.5 \%$. This figure explains the philosophy behind introducing this particular shape. Introducing more resistance to the flow path in the cross-tip direction forces the flow to change direction to a route of less resistance i.e., to turn with the main stream. In the triple-squealer shape, the leakage flow suffers from two successive sudden expansions followed by sudden contraction. The leakage flow first rolls against the pressure side squealer (PSS), then expands

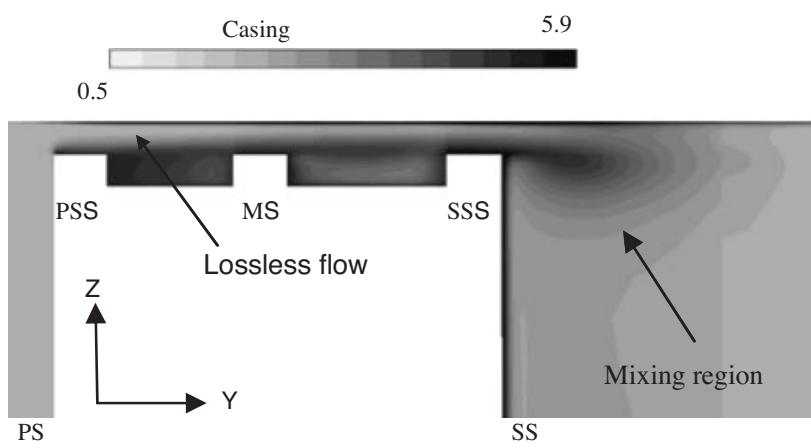

Fig. 9. Total pressure contours in plane with $x=0.5 C x$ (GDS ratio of $1.5 \%)$.

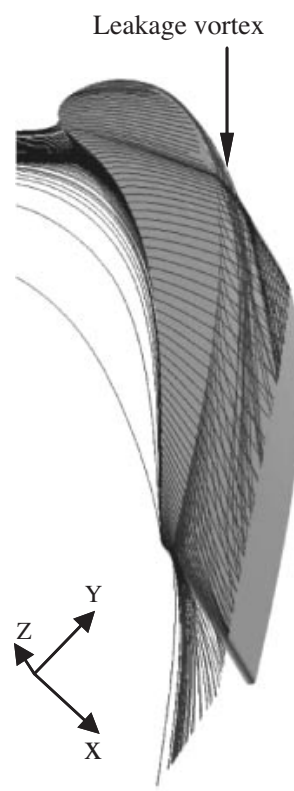

(a) Flat

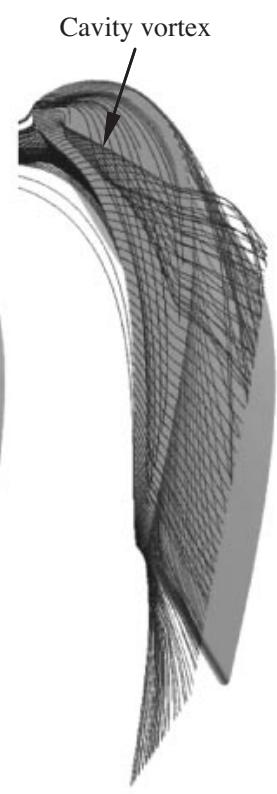

(b) Double squealer
Turning flow in second

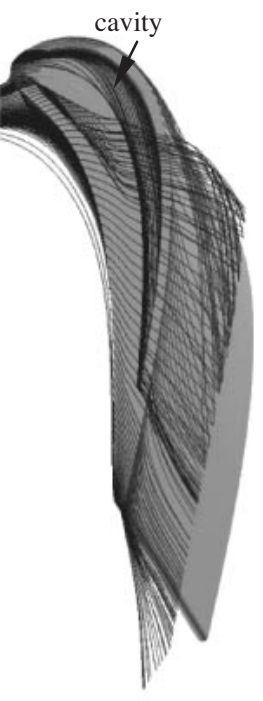

(c) Triple squealer GDS ratio of $=1.5 \%$

Fig. 10. 3D particle traces of near-tip flow for flat, and double and triplesquealer tips with GDS ratio of $1.5 \%$.

in the first cavity and loses some energy, then crosses the middle squealer (MS), expands again in the second cavity and loses more energy, then crosses the suction side squealer (SSS) and finally exits from the tip region and mixes with the main stream. The flow in both cavities is backward with different strengths.

Figure 9 shows the contours of the total pressure coefficient, $C p_{\mathrm{t}}$, at plane $x=0.5 C x$. The total pressure loss around the surfaces of the tip and casing, and in the cavities is high. Between the tip and casing, there is an inviscid, lossless flow, characterized by low total pressure loss. This inviscid flow suffers from mixing that reduces its thickness gradually across the tip. It seems that the flow near the casing is less affected by the squealers.

The particle traces of the near-tip flow are shown in Fig. 10 for flat, double and triple-squealer tips with GDS ratio of $1.5 \%$. Generally, the leakage flow emerging from the suction side rolls up, forming the leakage vortex shown in Fig. 10(a). For all cases with cavities, i.e., the double and 


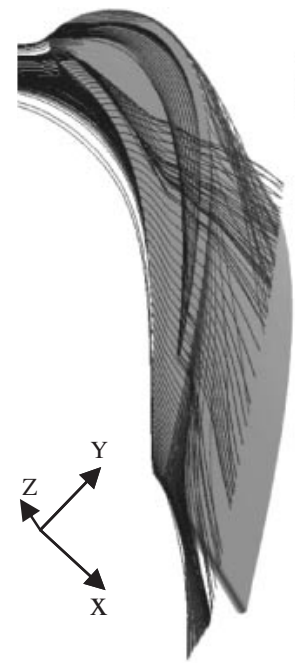

(a) GDS ratio $=0.75 \%$

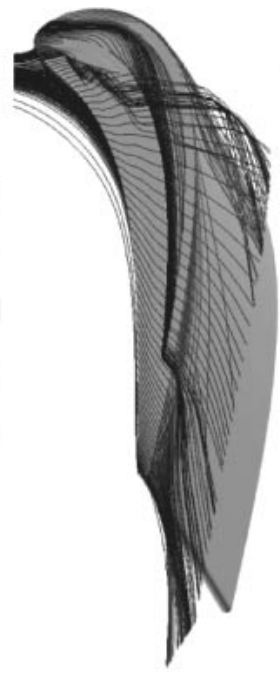

(b) $2.25 \%$

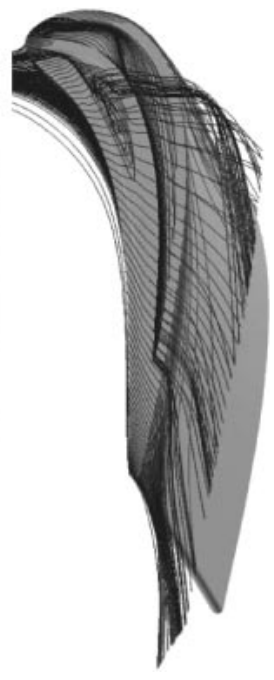

(c) $3 \%$
Fig. 11. 3D particle traces of near-tip flow for different values of cavity depth in triple-squealer tip.

triple-squealer cases, as a result of introducing cavities, the near-tip flow in the cavity rolls up, forming the vortex in the tip region shown in Fig. 10(b). This vortex in the tip region is called "cavity vortex" in this paper. Introducing the middle squealer divides the flow in the cavity into two parts. The near-tip flow in the first cavity (near the PSS), rolls up forming the cavity vortex. The cavity vortex crosses the middle squealer, explaining the existence of the relatively high velocity region on the middle squealer. Next, it crosses the second cavity where it gradually entrained the lossless flow and exits the tip between $x=0.48$ and $0.62 C x$, explaining the high velocity region on the suction-side squealer. The near-tip flow in the second cavity is mainly parallel to the suction-side squealer, because the middle squealer reduces the effect of the cavity vortex and provides a safe channel for the flow in the second cavity to flow parallel to the suction-side squealer. Direct comparison between Fig. 10(b) and (c) shows the effect of the middle squealer (MS) on the cavity vortex size, which is much smaller in the triple-squealer case than the double-squealer case.

\subsection{Comparison between triple-squealer cases}

Figure 11 shows the particle traces of the near-tip flow for the triple-squealer configuration for three values of GDS ratio: $0.75 \%, 2.25 \%$, and $3 \%$. Figure 11 indicates that the cavity vortex size and shape are a function of cavity depth. The possible reason for this vortex formation is the vorticity generated from the velocity gradient of the near-tip flow and the interaction between the flow entering the tip from the suction side and that entering from the first part of the pressure side close to the leading edge.

For the GDS ratio cases of $0.75 \%$ and $1.5 \%$, the cavity vortex is attached to the inner side of the pressure-side squealer. The cavity vortex size for the GDS ratio of $0.75 \%$ is slightly larger than for the GDS ratio of $1.5 \%$. This is attributed to the larger mass flow rate of the leakage

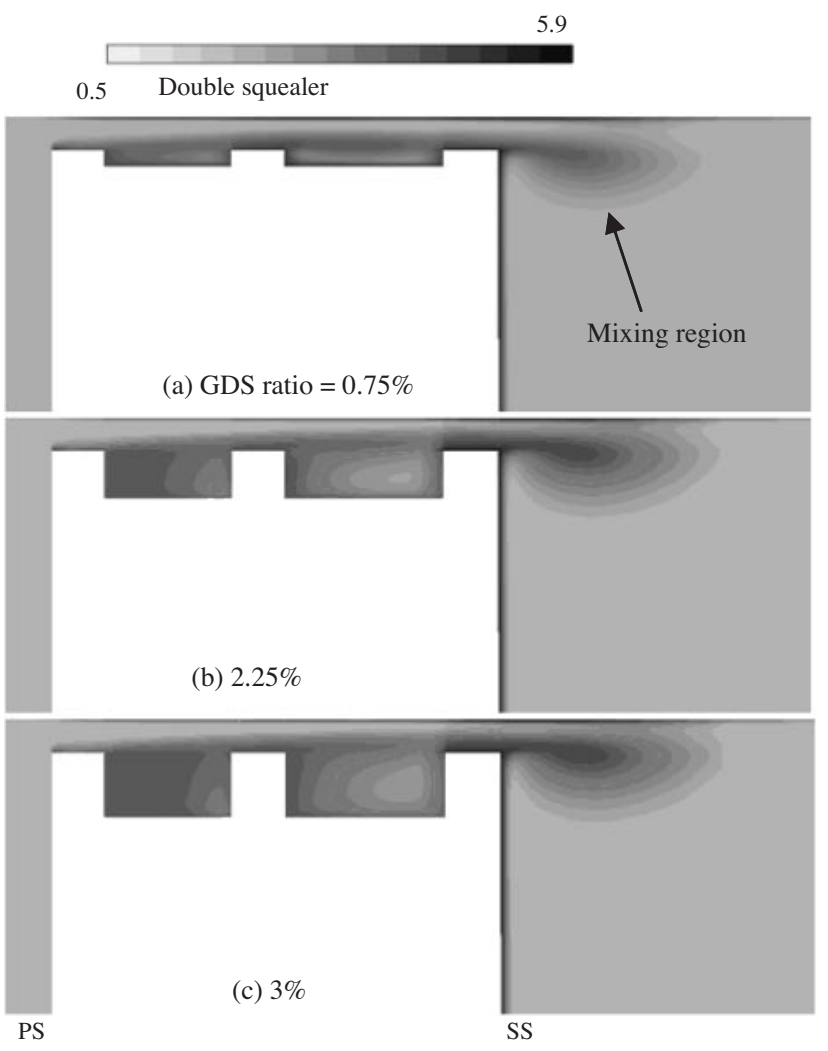

Fig. 12. Total pressure contours in plane with $x=0.5 C x$ for different values of cavity depth in triple-squealer tip.

flow in the $0.75 \%$ GDS ratio case. In the GDS ratio cases for $2.25 \%$ and $3 \%$, the cavity vortex is detached from the pressure-side squealer towards the middle squealer, where it crosses the second cavity (near suction-side squealer) quite normal to the camber line. At exit from the tip, it wraps around the leakage vortex formed previously.

Figure 12 shows the total pressure coefficient, $C p_{\mathrm{t}}$, at a plane of $x=0.5 C x$ for the triple squealer; three values of the GDS ratio are considered: $0.75 \%, 2.25 \%$, and $3 \%$. When the leakage flow exits the tip clearance, a mixing region is formed (marked in Fig. 12(a)). The area of the mixing region for a GDS ratio of $2.25 \%$ looks similar to that for a GDS ratio of $3 \%$, and smaller than that for a GDS ratio of $1.5 \%$ (Fig. 9). This is consistent with the results of leakage mass flow rate in Fig. 13, i.e., the size of mixing regions is proportional to the leakage mass flow rate. On the other hand, the area of the mixing region for a GDS ratio of $0.75 \%$ is smaller than that for other cases. This can be explained from Fig. 11(a) where the cavity vortex left the tip clearance at $x>0.5 C x$. For the flat-tip case shown in Fig. 5, the leakage flow is accompanied by a thick boundary layer, explaining the high- $C p_{\mathrm{t}}$ region near the tip. The shape of the mixing zone is elongated in the leakage flow direction because the leakage flow from the suction side has large kinetic energy. For the triple-squealer case with a GDS ratio of $1.5 \%$, there is a strong interaction between the leakage flow and flow in the cavities, which is marked by the high $C p_{\mathrm{t}}$ value. In the other cases for the triple squealer with a GDS ratio of $0.75 \%, 2.25 \%$, and 


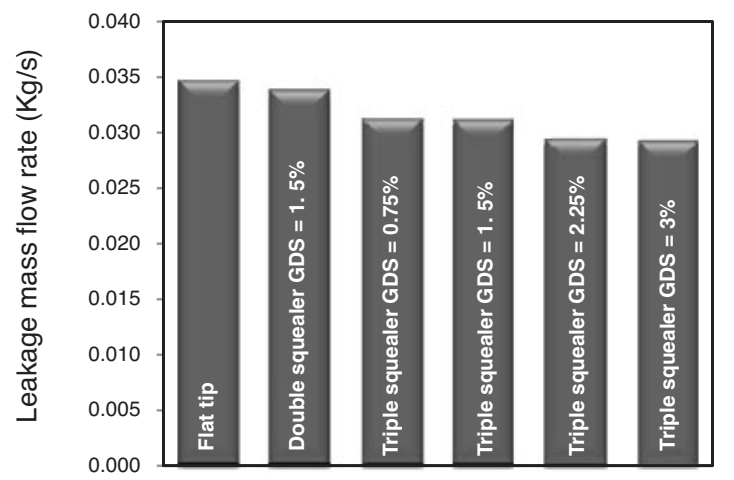

Fig. 13. Comparison of mass flow rate through tip region for different tip configurations.

$3 \%$, the interaction strength is significantly reduced especially in the second cavity (close to suction side). This directs our next research towards the best design parameters for the cavities.

Figure 13 shows the integral of the mass flow rate through the tip region for three kinds of tip configuration: flat, double squealer, and triple squealer. For the triple squealer, four values of the GDS ratio are considered: $0.75 \%, 1.5 \%$, $2.25 \%$, and $3 \%$. From the figure, the variation of mass flow rate through the tip region is not in a linear relation with the variation in cavity depth. By introducing the double squealer, the mass flow rate of leakage flow through the suction side is reduced by $2.32 \%$ compared to the baseline case (flat tip). For the triple squealer, it is $9.53 \%, 10.1 \%, 15.25 \%$, and $15.69 \%$ for GDS ratios of $0.75 \%, 1.5 \%, 2.25 \%$, and $3 \%$ respectively. The case of the triple squealer with a GDS ratio of 3\% gives the highest reduction in mass flow rate through the tip region. While that for a GDS ratio of $0.75 \%$ gives the lowest reduction between the tested triple-squealer cases. However, the difference in the leakage mass flow rate between the triple-squealer cases for a GDS ratio of $2.25 \%$ and $3 \%$ is not significant. It is worth noting that the reduction in leakage mass flow rate in the triplesquealer case for a GDS ratio of 3\% is 1.65 times that for a GDS ratio of $0.75 \%$, which is 4.1 times that for the double squealer.

The mass averaged total pressure coefficient is defined as:

$$
\xi=\frac{\int C p_{\mathrm{t}} \rho u \delta A}{\int \rho u \delta A},
$$

where, $u$ is the velocity and $A$ the cross-sectional area. $\xi$ at the axial plane $20 \%$ chord downstream (plane $\mathrm{P}$ in Fig. 2(b)), is shown in Fig. 14 indicating that the flat tip has the highest $\xi$, which is consistent with the results of the leakage mass flow rate where the flat tip has the largest leakage mass flow rate. On the other hand, the double squealer has the lowest $\xi$. Although the leakage mass flow rate is smaller for the triple squealer with a GDS ratio of $1.5 \%$ than for the double squealer, as shown in Fig. 13, $\xi$ for the triple squealer is slightly larger than for the double squealer. This difference, although small compared to that in the flat tip, can be explained by two factors. First, the

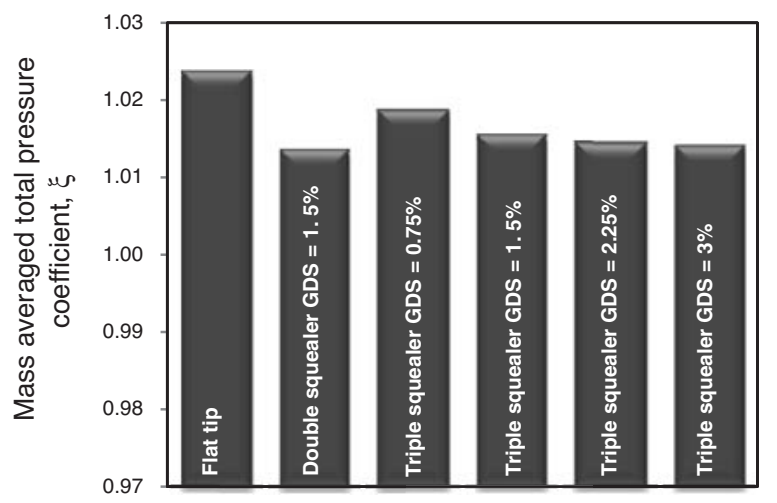

Fig. 14. Mass-averaged total pressure coefficient.

increase in the internal loss, which is the total pressure loss, occurs at the tip clearance due to the middle squealer and cavities as shown in Fig. 9. Second, the chord-wise length of the cavity in the double squealer is longer than in the triple squealer as shown in Fig. 1(c and d). Hence, the rear part of the triple squealer affects the leakage flow similarly to the flat tip. Figure 14 also shows that $\xi$ for the triple-squealer cases decreases with increase in GDS ratio. However, the decrease in $\xi$ after the GDS ratio of $2.25 \%$ is smaller than before. Among calculated triple-squealer cases, the triple-squealer case with a GDS ratio of 3\% has the lowest $\xi$.

\section{Conclusion}

A new blade tip shape called triple squealer is proposed. This shape is based on the conventional double squealer by dividing the cavity into two parts by using a third squealer along the blade camber line. Four cases for the ratio of groove depth to span (GDS ratio): $0.75 \%, 1.5 \%, 2.25 \%$, and $3 \%$, which correspond to $50 \%, 100 \%, 150 \%$, and $200 \%$ of the tip clearance to span ratio, respectively, were used to investigate the effects of GDS ratio on the performance of the triple squealer. The following conclusions were drawn:

- The triple squealer successfully reduces leakage mass flow rate by more than the double squealer for the same GDS ratio. However, the total pressure loss is higher for the triple squealer than for the double squealer.

- The triple-squealer case for a GDS ratio of 3\% gives the highest reduction in leakage mass flow rate by $15.69 \%$ that of the flat-tip case. It also achieves the lowest mass averaged total pressure loss among the triple-squealer cases.

-In the triple squealer, both the leakage mass flow rate and the total pressure loss are inversely proportional to the increase in GDS.

-Further systematic assessment is needed to evaluate the performance of the triple-squealer shape, starting with studying the effect of the triple-squealer shape on stage efficiency followed by heat transfer and external cooling. 


\section{Acknowledgments}

The first author is thankful to the Egyptian Government, Ministry of Higher Education for providing him the scholarship to have Ph D. This work was supported by a Grant-in-Aid for the 21 st Century COE Program "Frontiers of Computational Sciences" from Ministry of Education, Culture, Sports, Science and Technology, Japan. The authors would like to thank Prof. Igor Men'shov for his valuable advices and guidance during his stay and visit to Nagoya University, and Mr. M. El-Gendi, Dr. M. Jones, Dr. Kitamura, and Mr. Hirose for their discussion and support.

\section{References}

1) Booth, T. C.: Importance of Tip Leakage Flow in Turbomachinery Design, Tip Clearance Effects in Axial Turbomachinery, VKI Lecture Series, 1985-05, Von Karman Institute for Fluid Dynamics, Belgium, 1985.

2) Sjolander, S. A.: Physics of Tip Clearance Flows, Part I, Secondary and Tip-Clearance Flows in Axial Turbines, VKI Lecture Series, 1997-01, Von Karman Institute for Fluid Dynamics, Belgium, 1997.

3) Bunker, R. S.: Axial Turbine Blade Tip: Function, Design, and Durability, J. Propul. Power, 22 (2006), pp. 271-285.

4) Azad, G. S., Han, J. C., Bunker, R. S. and Lee, C. P.: Effect of Squealer Geometry Arrangement on a Gas Turbine Blade Tip Heat Transfer, J. Turbomach., 124 (2002), pp. 452-459.

5) Kwak, J. S., Ahn, J., Han, J. C., Lee, C. P., Bunker, R. S., Boyle, R. and Gaugler, R.: Heat Transfer Coefficients on the Squealer Tip and Near Squealer Tip Regions of a Gas Turbine Blade with Single or Double Squealer, J. Turbomach., 125 (2003), pp. 778-787.

6) Saxena, V., Nasir, H. and Ekkad, S. V.: Effect of Blade Tip Geometry on Tip Flow and Heat Transfer for a Blade in a Low-Speed Cascade, J. Turbomach., 126 (2004), pp. 130-138.
7) Prakash, C., Lee, C. P., Cherry, D. G., Doughty, R. and Wadia, A. R.: Analysis of Some Improved Blade Tip Concepts, J. Turbomach., 128 (2006), pp. 639-642.

8) Saha, A. K., Acharya, S., Bunker, R. and Prakash, C.: Blade Tip Leakage Flow and Heat Transfer with Pressure-Side Winglet, Int. J. Rotating Machinery, 2006 (2006), pp. 1-15.

9) Mischo, B., Behr, T. and Abhari, R. S.: Flow Physics and Profiling of Recessed Blade Tips: Impact on Performance and Heat Load, J. Turbomach., 130 (2008), 021008, 8 pages.

10) Moore, J. and Tilton, J. S.: Tip Leakage Flow in a Linear Turbine Cascade, J. Turbomach., 110 (1988), pp. 18-26.

11) Moore, J., Moore, J. G., Henry, G. S. and Chaudhry, U.: Flow and Heat Transfer in Turbine Tip Gaps, J. Turbomach., 111 (1989), pp. 301-309.

12) Ameri, A. A. and Steinthorsson, E.: Analysis of Gas Turbine Rotor Blade Tip and Shrouded Heat Transfer, NASA CR-198541, 1996.

13) Ameri, A. A. and Steinthorsson, E.: Prediction of Unshrouded Rotor Blade Tip Heat Transfer, NASA CR-198542, 1996.

14) Yang, H., Acharya, S., Ekkad, S. V., Parakash, C. and Bunker, R.: Flow and Heat Transfer Predictions for a Flat Tip Turbine Blade, Proceedings of ASME TURBO EXPO 2002, June 3-6, Amsterdam, The Netherlands.

15) Timko, L. P.: Energy Efficient Engine High Pressure Turbine Component Test Performance Report, NASA CR-168289, 1984.

16) Kitamura, K., Men'shov, I. and Nakamura, Y.: Shock/Shoch and Shoch/Boundary-Layer Interactions in Two-Body Configurations, 35th AIAA Fluid Dynamics Conference and Exhibit, 6-9, June 2005, Toronto, Canada, AIAA-2005-4893.

17) Li, D., Men'shov, I. and Nakamura, Y.: Detached-Eddy Simulation of Three Airfoils with Different Stall Onset Mechanisms, J. Aircraft, 43 (2006), pp. 1014-1021.

18) Sjolander, S. A. and Amrud, K. K.: Effects of Tip Clearance on Blade Loading in a Planar Cascade of Turbine Blades, J. Turbomach., 109 (1987), pp. 237-245. 